\title{
Einführung und Begleitung von Peer-Review-Verfahren seitens der Bundesärztekammer
}

\section{G. Jonitz}

Qualitätsmanagement

Schlüsselwörter

- Peer Review

Klinische Audits

Evaluation

$\checkmark$ Qualitätsmanagement

Qualitätsverbesserung

- Kollegialer Dialog

Versorgungsqualität

Keywords

medical peer review

clinical audits

Oevaluation

quality management

quality improvement

collegial reflection

quality of care
Institut

Bundesärztekammer, Berlin

Bibliografie

DOI 10.1055/s-0031-1286083

Dtsch Med Wochenschr 2011;

136: S54 - (c) Georg Thieme

Verlag KG Stuttgart · New York .

ISSN 0012-0472

Korrespondenz

Dr. med. Günther Jonitz

Bundesärztekammer

Herbert-Lewin-Platz 1

10623 Berlin

Tel. 030/400456-437

eMail info@baek.de
Peer Review ist eine klassische Methode der unmittelbaren, fachlich höchstwertigen, konkreten und konstruktiven Qualitätssicherung in der Medizin. Peers sind dabei Ärztinnen und Ärzte, die als anerkannte Experten ihres jeweiligen Fachgebietes mit den Abläufen einer Klinik bestens vertraut und als ärztliche Kollegen anerkannt sind.

Sie analysieren - gemeinsam mit den Ärzten der zu begutachtenden Einrichtung vor Ort - systematisch Prozesse und Strukturen auf mögliche Optimierungspotenziale hin, sodass ein direkter Praxisbezug durch das Verfahren selbst gewährleistet wird.

Vor dem Hintergrund, dass Qualitätssicherung heutzutage von vielen Ärztinnen und Ärzten als lästige, fremdbestimmte Tätigkeit empfunden wird, die sie vom eigentlichen ärztlichen Handeln abhält, nimmt es nicht Wunder, dass das Peer-Review-Verfahren als unbürokratisches, flexibles und auf den kollegialen Austausch fokussiertes Instrument zur Qualitätsförderung zunehmend an Attraktivität und Akzeptanz innerhalb der Ärzteschaft gewinnt und von den Landesärztekammern und der Bundesärztekammer gefördert wird.

Kernstück dieses qualitativen, auf Entwicklung gerichteten Evaluationsverfahrens ist der Besuch des Peer-Review-Teams vor Ort. Die zuvor systematisch in einer Selbst- und einer Fremdbewertung erhobenen Daten zur Versorgungsqualität der Patienten werden im kollegialen Dialog reflektiert. Dabei liegt der Fokus auf der Identifizierung von Verbesserungspotenzialen und der gemeinsamen Erarbeitung von Lösungsansätzen. Voraussetzung für den Erfolg des Reviews ist eine vertrauensvolle, von Schuldzuweisung bzw. Rechtfertigungsnot freie Atmosphäre, in der die Reviewer und die Kollegen der begutachteten Einrichtung auf Augenhöhe reflektieren, wie das eigene ärztliche Handeln sowie Abläufe noch optimaler gestaltet werden können. Dabei wird stets der gesamte Behandlungsprozess des Patienten beleuchtet - eine Herangehensweise, die auch zu einer stärkeren Integration aller beteiligten Fachdisziplinen führt sowie deren Kommunikation untereinander intensiviert.

Die aktuell in Deutschland praktizierten PeerReview-Verfahren wurden freiwillig von Ärzten für Ärzte entwickelt. Die initialen Impulse gingen von der Initiative Qualitätsmedizin $\left(\mathrm{IQ}^{\mathrm{M}}\right)$ aus. Erste Erfahrungen aus den von der Bundesärztekammer evaluierten Pilot-IQ ${ }^{\mathrm{M}}$-Peer-Re- views bescheinigen dem Verfahren einen hohen Praxisbezug und - durch das zeitnahe Feedback - einen sofort spürbaren Nutzen. Als positive Nebeneffekte werden ein Zuwachs der professionellen Autonomie auf beiden Seiten und die Beschleunigung von Veränderungen berichtet. Die Fach- und Berufsgruppen werden stärker integriert und motiviert, vor allem da sie Qualitätsentwicklung als selbstbestimmt wahrnehmen.

Gemeinsam mit den Landesärztekammern unterstützte die Bundesärztekammer von Anfang an die Pilot-Peer-Reviews von $\mathrm{IQ}^{\mathrm{M}}$, indem sie sie durch Experten begleitend beobachten und evaluieren ließ. Der sich bereits zu Beginn der Pilotphase abzeichnende Qualifizierungsbedarf für die Peers führte zur Entwicklung des Curriculum „Ärztliches Peer Review“ [1], das im Februar 2011 vom Vorstand der Bundesärztekammer verabschiedet wurde.

Seitdem besteht eine hohe Nachfrage nach entsprechenden Qualifizierungsangeboten für Peers. Im April 2011 fand die bundesweit erste PeerReview-Schulung an der Ärztekammer Berlin statt.

Das Curriculum „Ärztliches Peer Review“ ist auf der Website der Bundesärztekammer einsehbar: www.baek.de.

Autorenerklärung: Als Autor des o.g. Artikels erkläre ich, dass ich keine finanziellen Verbindungen mit einer Firma habe, deren Produkt in dem Artikel eine wichtige Rolle spielt (oder mit einer Firma, die ein Konkurrenzprodukt vertreibt).

\section{Literatur}

1 Bundesärztekammer (BÄK). Curriculum Ärztliches Peer Review, 1. Auflage Berlin: 2011 